\title{
Correlation of Health Literacy with posttraumatic growth of patients with \\ cancer- A cross-sectional study in Tehran
}

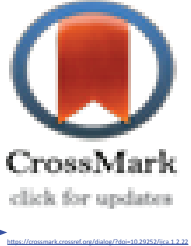

\author{
Bolourchifard $\mathrm{F}^{1}$, Rasouli $\mathrm{M}^{2}$, Ramezani $\mathrm{S}^{3 *}$
}

1- Assistant Professor, Department of Nursing, Faculty of Nursing and Midwifery, Shahid Beheshti University of Medical Sciences, Tehran, Iran.

2- Professor Department of Nursing, Faculty of Nursing and Midwifery, Shahid Beheshti University of Medical Sciences, Tehran, Iran.

3- M.Sc. in Nursing, Department of Nursing, Student Research Committee of the Faculty of Nursing and Midwifery, Shahid Beheshti University of Medical Sciences, Tehran, Iran .

Corresponding Author: Ramezani S, M.Sc. in Nursing, Department of Nursing, Student Research Committee of the Faculty of Nursing and Midwifery, Shahid Beheshti University of Medical Sciences, Tehran, Iran.

Email:ramezanisalar1991@gmail.com

Received: 11 December 2018

Accepted: 29 May 2019

\section{Abstract}

Introduction: Post-Traumatic Growth refers to personal and psychological changes that have been made after a severe incident and the result of a person's struggle against the incident is stressful. This study aimed to investigate the correlation between post-traumatic post-traumatic incidence and health literacy in in-patient patients in educational hospitals of Shahid Beheshti University of Medical Sciences.

Method: In this analytical-cross-sectional study, 384 patients with cancer were selected in hospitals affiliated to Shahid Beheshti University of Medical Sciences. Data were collected by Health Literacy and Post-Traumatic Growth questionnaires. Their validity and reliability were confirmed. Data analysis were used by descriptive statistics, independent t-test, Pearson correlation and ANOVA in SPSS 22 software.

Results: According to findings, the lowest and the most average of Post-Traumatic Growth scores were related to dimensions of new possibilities (2.04) and spiritual changes (3.84). Also, the lowest and highest mean of Health Literacy scores were in reading area (2.99) and comprehension domain (4.06) respectively. The average total score of Post-Traumatic Growth and Health Literacy was 3.1 and 3.68, respectively. There was a significant and positive correlation between Post-Traumatic Growth and Health Literacy in patients with cancer.

Conclusion: Due to the link between Post-Traumatic Growth and Health Literacy, it is necessary to provide quantitative and qualitative counseling programs and improve the quality of health care and improve patientprovider communication, to provide good conditions for patients to better understand and adapt to their circumstances.

Key words: Post-Traumatic Growth, Health Literacy, Cancer Patients, Educational Hospital.

\begin{tabular}{|l|l|}
\hline \multicolumn{3}{|c|}{ Access this article online } \\
\hline Website: \\
www.ijca.ir
\end{tabular}




\section{همبستى رشد يِ از سانحه باسواد سلامت در بيمار ان مبتلا به سرطان، يك مطالعه مقطعى در تهران}

فريبا بلورجى فرد'، مريم رسولى"، سالار رمضانى" "

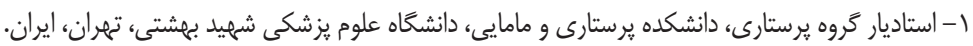

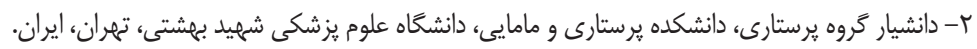

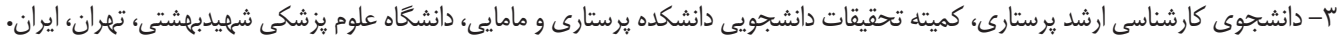

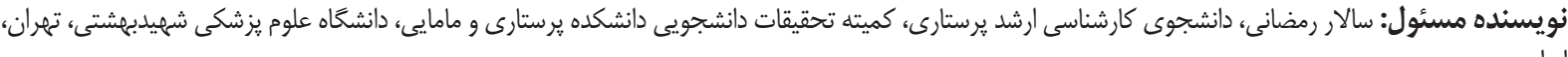

ايميل: ramezanisalar1991@gmail.com

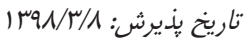

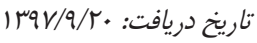

مقدمه: رشد يس از سانحه، به تغييرات شخصى و روانشناختى مثبتى كَته مى شود كه يس از وقوع يكى حادثه ايجاد شده و نتيجهى مبارزه

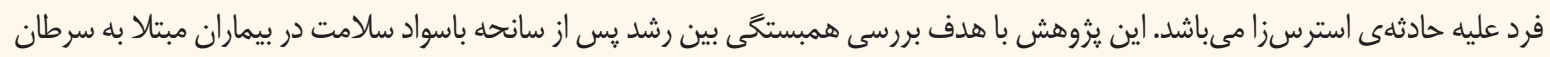

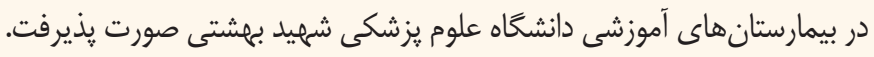

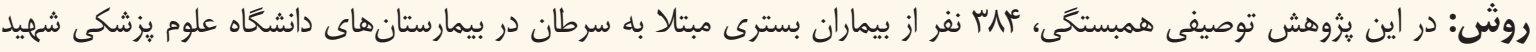

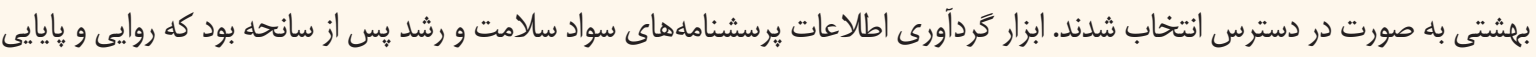

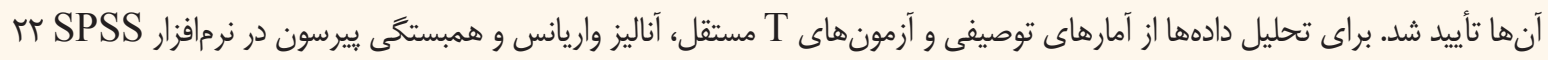
| استفاده شد.

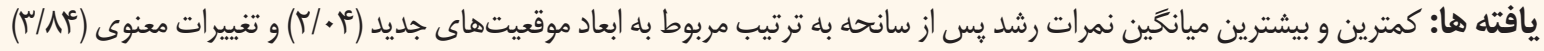

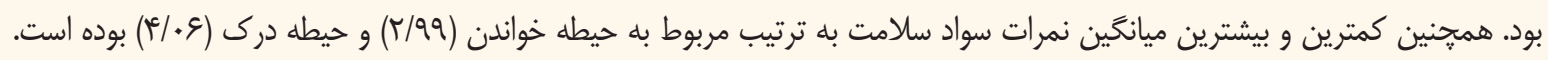

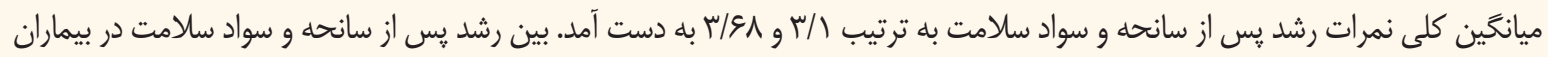
مبتلا به سرطان همبستخى مثبت وجود داشت.

نتيجه كَيرى: به دليل وجود همبستخى بين رشد يس از سانحه و سواد سلامت ضرورى است با ارائه كمى و كيفى برنامههاى مشاوره و

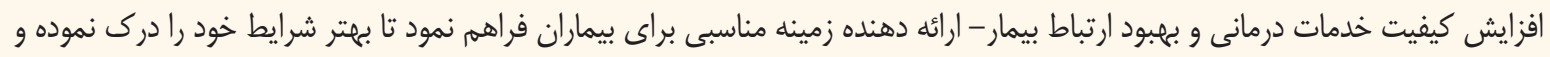
با آن سازكًار شوند. كليدوازه ها: رشد يس إز سانحه، سواد سلامت، بيماران مبتلا به سرطان، بيمارستان هاى آموزشى.

ممكن است در اثر تجربه ابتلا به اين بيمارى، يِيامدهاى مثبتى را

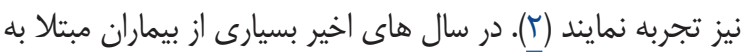

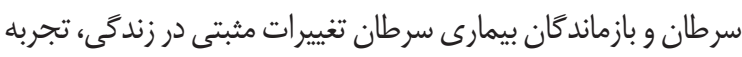

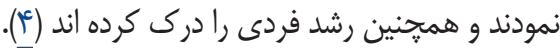

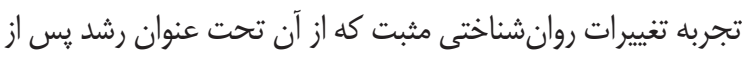

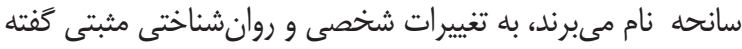

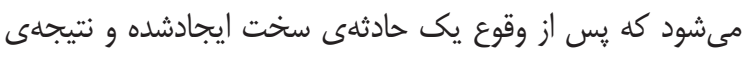

مبارزه فرد عليه اين حادثهى استرسزا مىباشد (ه، كان).

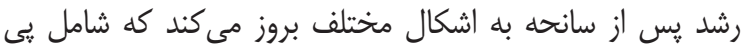

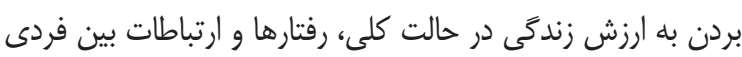
بروز بيمارى هاى تهديد كننده زندكى مانند سرطان منجر به افزايش

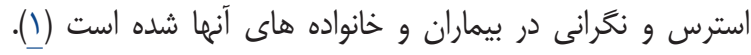

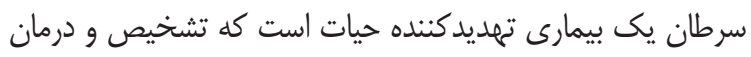

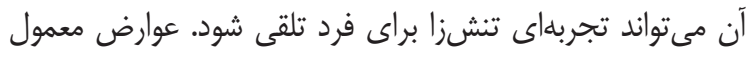

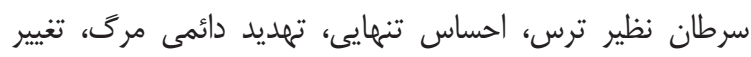

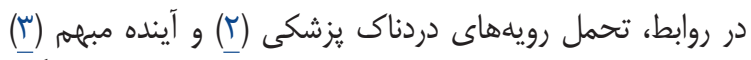

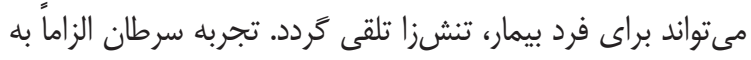

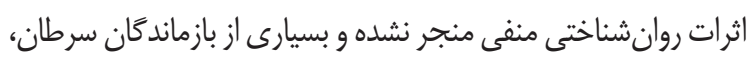




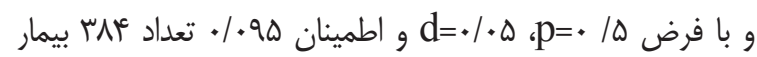

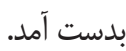

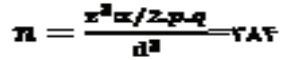

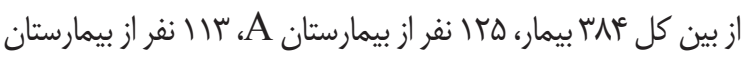
B كه تخصيص نمونه ها بر اساس تعداد تخت در هر بيمارستان صورت

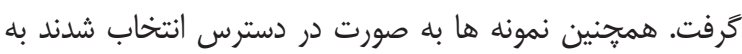

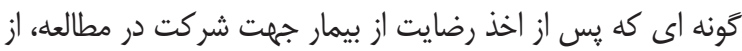

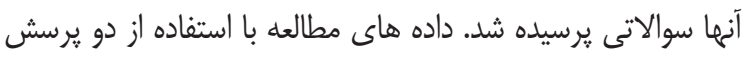

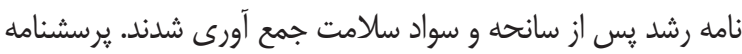

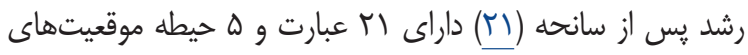

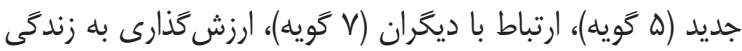

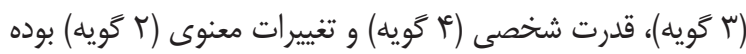

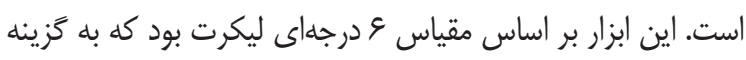

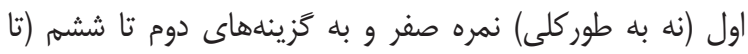

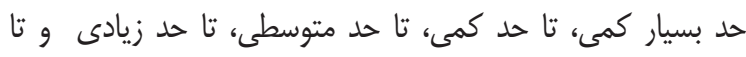

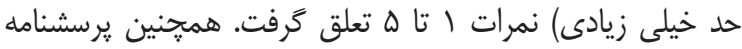

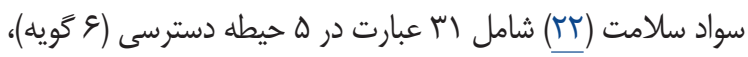

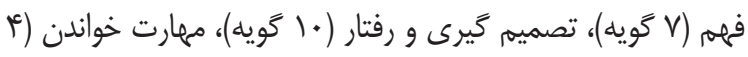

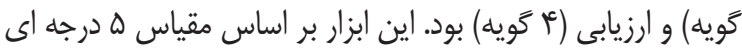

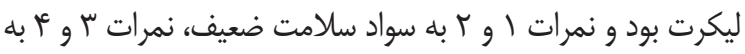

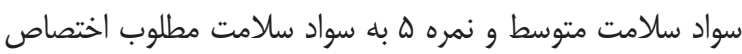

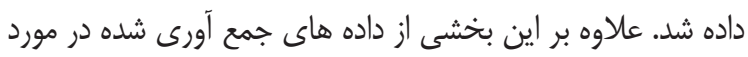

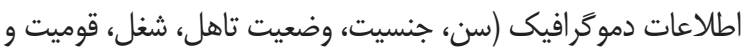
بيمه درمانى) بيمار ان بوده است.

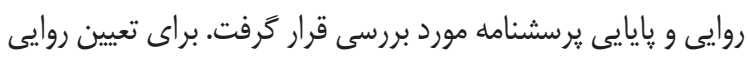

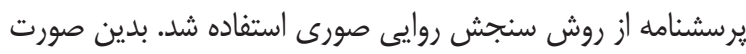

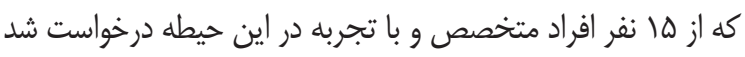

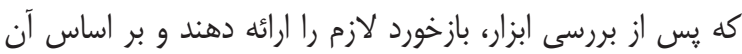

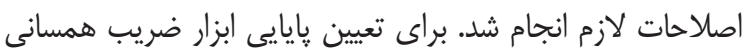

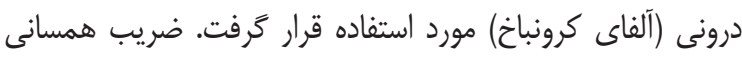

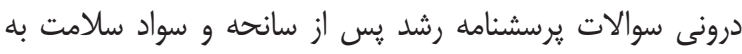

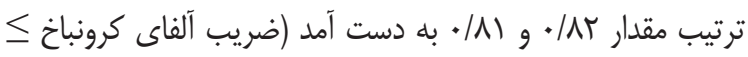

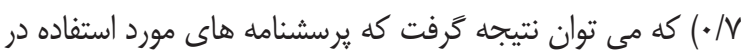

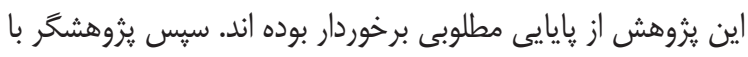

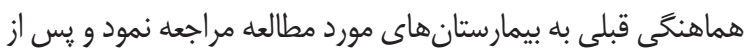

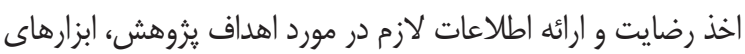

معنادارتر، احساس قوىتر شدن، تغيير در اولويتها و غنىتر شدن

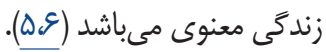

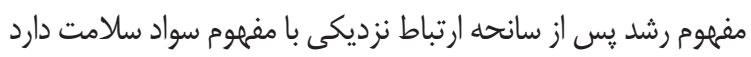

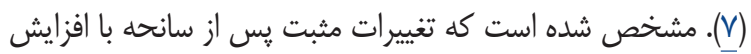

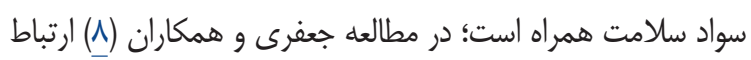
مستقيم و معنى دارى بين بعد معنوى مذهب و سواد سلامت مشاهده

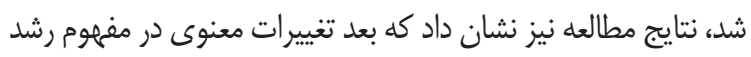

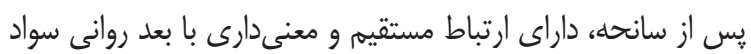

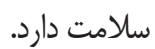
سواد سلامت عبارت از ميزان ظرفيت فرد براى كاد كسب، تفسير،

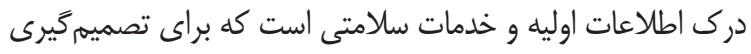

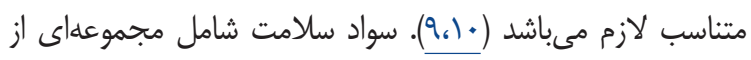

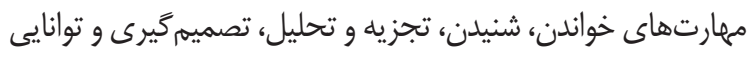

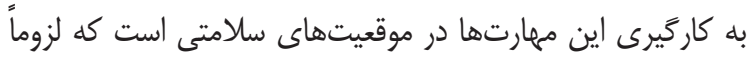
به سالهاى تحصيل يا توانايى خواندن عمومى برنمى كردد (11).

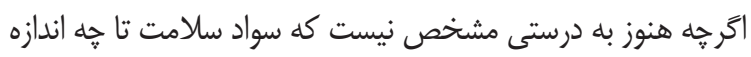

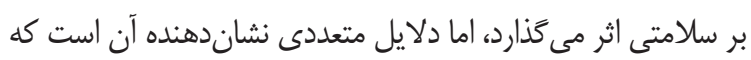
بسيارى از نتايج نامطلوب وابسته به سلامتى، در اثر سواد سلامت

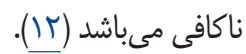
افراد داراى سواد سلامتى اندى، كمتر مىتوانند اطلاعات نوشتارى

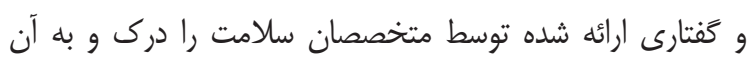

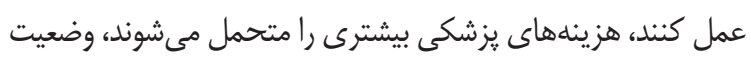
سلامت ضعيفترى دارند، ميزان بسترى شدن و استفاده از خدمات

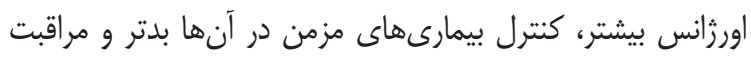

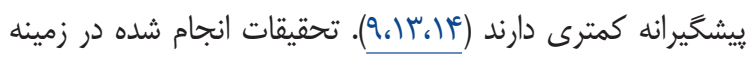

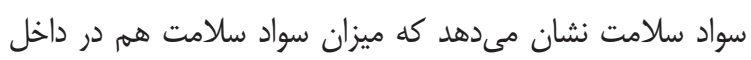

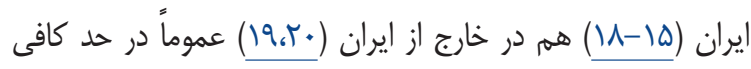
نمى ايراند. با توجه به اينكه تاكنون مطالعهاى در زمينه بررسى ارتباط سواد

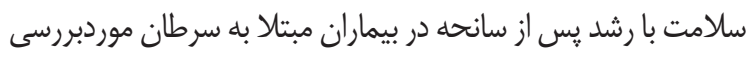

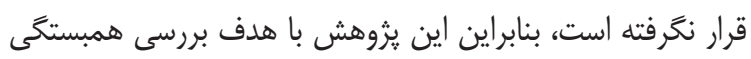

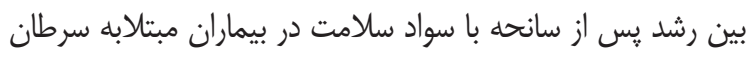
بسترى در بيمارستانهاى آموزشى دانشخاه علوم يزشكى شئ شهيد

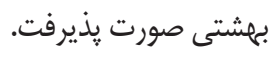

\section{روش مطالعه}

روش يزوهش توصيفى از نوع همبستخى مى باشد. جامعه آمارى اين

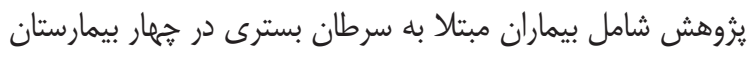

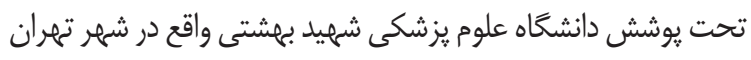

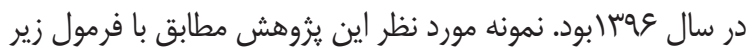


معنوى (ז/N/N) و كمترين ميانخين مربوط به حيطه موقعيتهاى

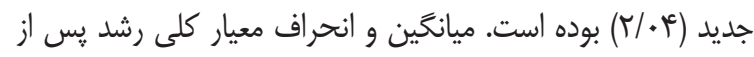

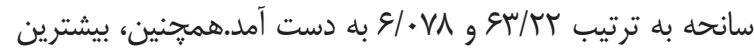

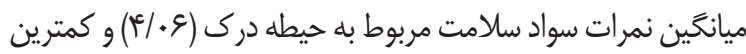

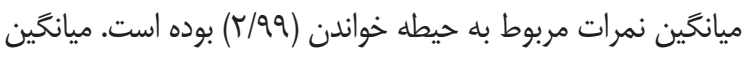

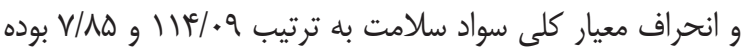

است.

بر اساس يافتههاى (جدول ()، ارتباط معنى دارى بين متغيرهاى مورد مطالعه با متغير رشد يس از سانحه يافت نشد. در اين مطالعه سطح

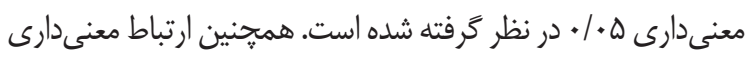
بين متغير گروه سنى و سواد سلامت بيماران وجود داشت. بدين

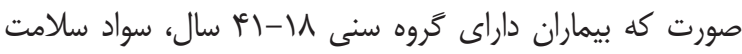

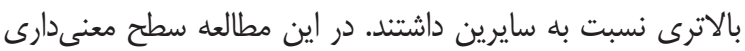

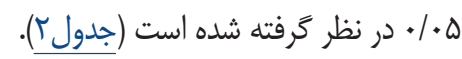

كَرداورى اطلاعات در اختيار بيماران قرار داده شد. يثوهشگر در ضمن تكميل يرسشنامه ها توسط بيماران حضور داشت و در صورت نياز

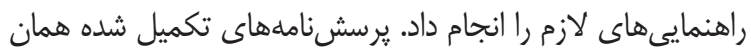

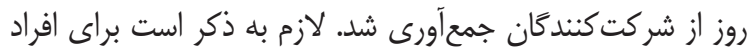

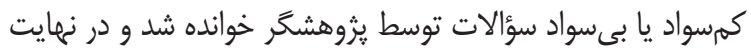
داده هاى مطالعه با استفاده از روش هاى آمار توصيفى، آناليز واريانس

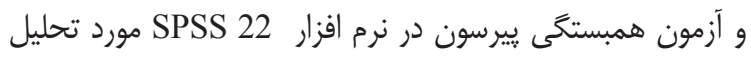

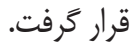

\section{يافته ها}

بر اساس يافته هاى اين مطالعه بيماران به جنسيت زن (\%عهاه)، متاهل

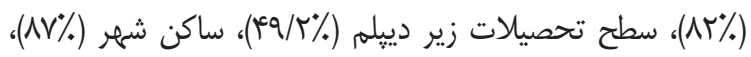

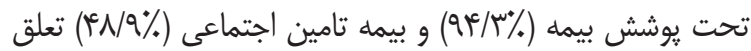
داشتند. بيشترين ميانگين نمرات رشد پِ از سانحه مربوط به حيطه تغييرات

جدول (ا: بررسى رابطه بين رشد پس از سانحه بيماران مبتلا به سرطان با متغيرهاى دموكر افيك

\begin{tabular}{|c|c|c|c|c|}
\hline P-Value & انحراف معيار & ميانَحين & 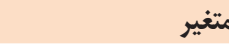 & \\
\hline$\cdot, V T I$ & $\begin{array}{l}q, 4) \\
0, \wedge 9\end{array}$ & מז, ז', & زن & جنسيت \\
\hline$\cdot$, rA. & $\begin{array}{l}9, \cdot 9 \\
8,4 c\end{array}$ & 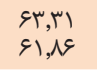 & ندارد & يوشش بيمه \\
\hline . & $\begin{array}{l}8, r \\
\Delta, \cdot 1\end{array}$ & 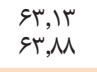 & روستا & محل سكونت \\
\hline • & $\begin{array}{c}8, \mu \\
g \\
0, \xi \mu\end{array}$ & $\begin{array}{l}\text { ST, ID } \\
\text { ST,qr } \\
\text { St,ir }\end{array}$ & $\begin{array}{l}|\lambda-| q \mid \\
F r-90 \\
99-19\end{array}$ & گروه سنى \\
\hline וחוז, & $\begin{array}{l}\Delta, \Lambda \\
\Delta, \Lambda \\
V, r \\
9, \Delta \\
9, q\end{array}$ & 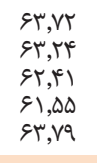 & 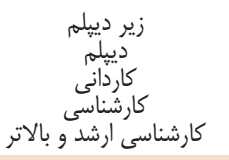 & سطح تحصيلات \\
\hline ( & $\begin{array}{l}\varepsilon, r \\
\varepsilon, \cdot r \\
0, r\end{array}$ & $\begin{array}{l}\text { st,q } \\
\text { st,is } \\
99, \wedge r\end{array}$ & ساير(بيوه-مطلـله مجله & وضعيت تأهل \\
\hline ש & $\begin{array}{l}\Lambda, r \omega \\
\Lambda, \bullet q \\
V, \Lambda 1 \\
q, 9 q\end{array}$ & $\begin{array}{l}11 r, q \\
11 r, 4 \\
119,+\wedge \\
11 r, r r\end{array}$ & خبازنشارته & وضعيت شغلى \\
\hline • & $\begin{array}{l}\Delta, H Y \\
9, Y Y \\
\Delta, V G \\
0, V \\
q, V G\end{array}$ & 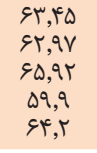 & 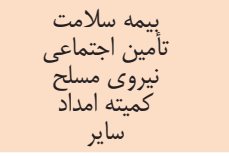 & نوع بيمه \\
\hline$\cdot, 1 \Delta \xi$ & $\begin{array}{l}q, r \\
0, r v \\
r, \Psi \wedge \\
V, i q \\
\Delta, \Delta r\end{array}$ & 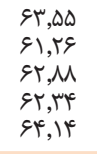 & ترك & قوميت \\
\hline$\cdot, \Delta \vee \wedge$ & $\begin{array}{l}\Delta, \vee \\
9, \wedge \\
r, q \\
0, r \\
0,9 \\
9,4 \\
4, \Lambda\end{array}$ & 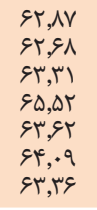 & 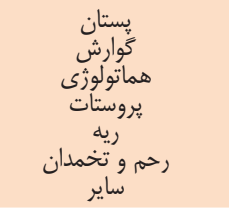 & نوع سرطان \\
\hline$\cdot, \Delta \Leftarrow \Delta$ & - & - & ن تشخيص & مدت \\
\hline
\end{tabular}




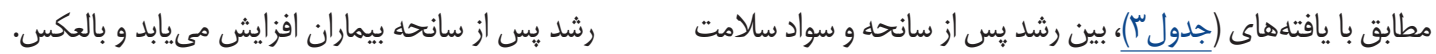

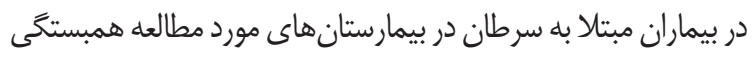

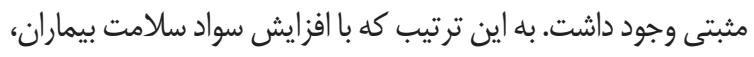
جدول ז: بررسى رابطه بين سواد سلامت بيماران مبتلا به سرطان با متغيرهاى دموكرافيك

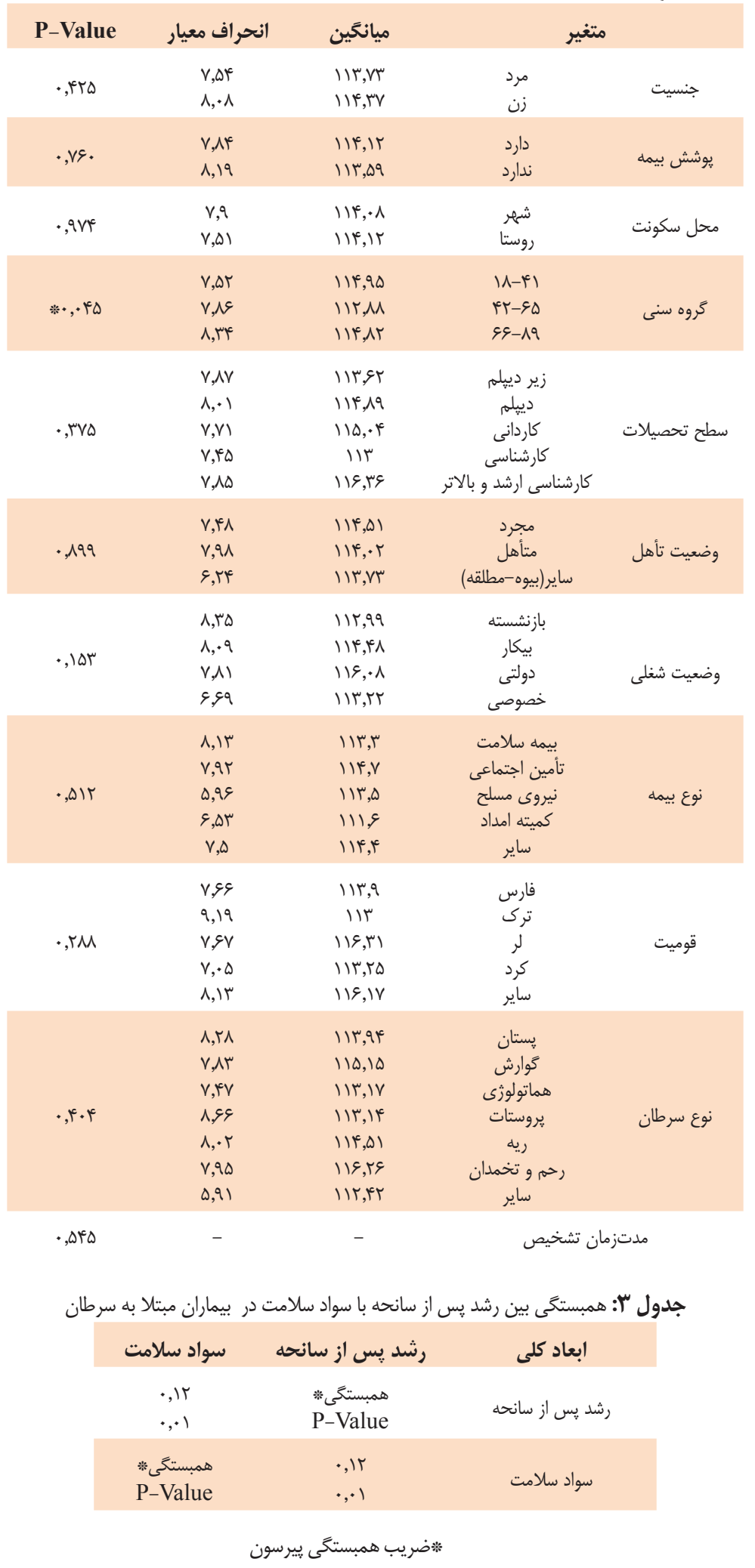


انجام شده در كشور نپال همراستا با يافتههاى اين مطالعه است

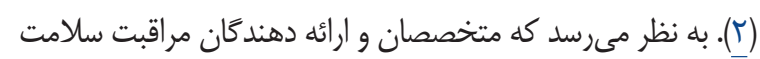

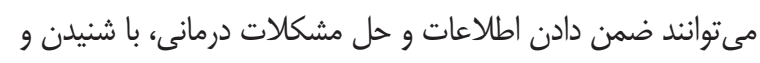

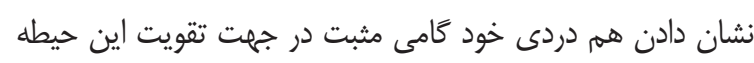

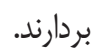
يافتهاى ديكر نشان دادند كه حيطه ارزش گذارى به زندگى نيز

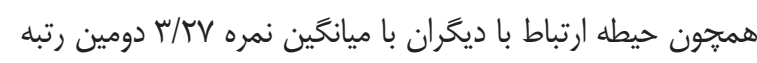

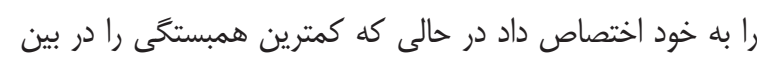

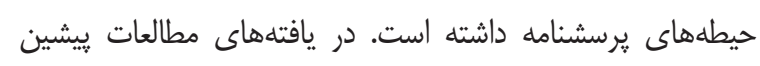

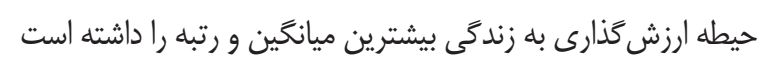

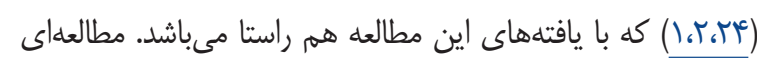

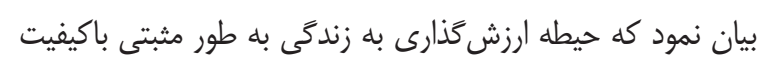

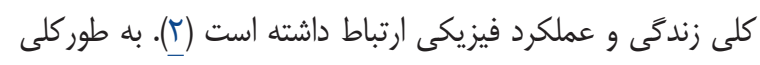

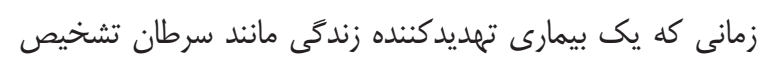

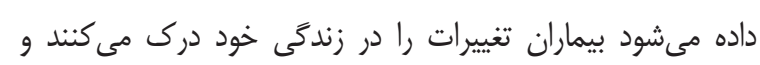

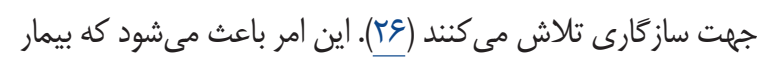

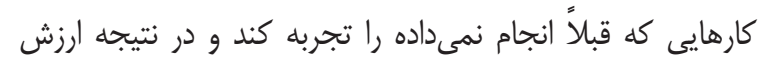

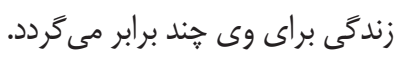
مطابق با يافتههاى اين مطالعه حيطه تغييرات معنوى با ميانكَين نمره

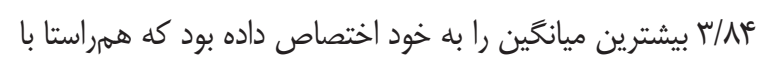

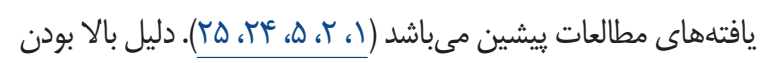

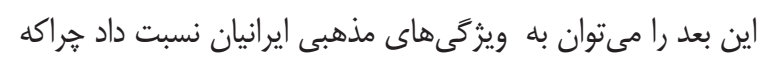

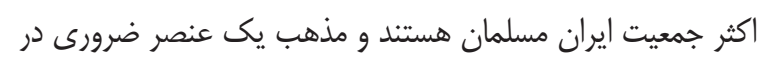

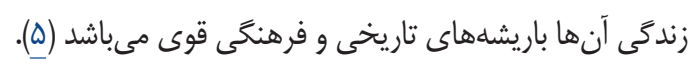

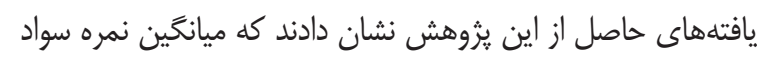

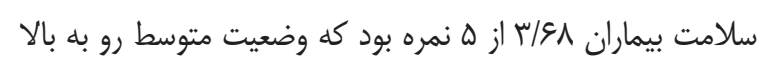
را نشان مىدهد. شواهد نشان مىدهند كه جهت بهببود دسترسى إنى

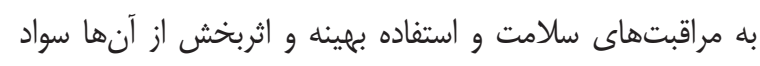

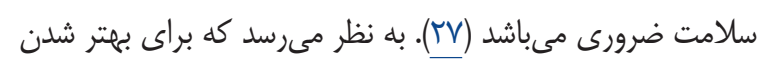

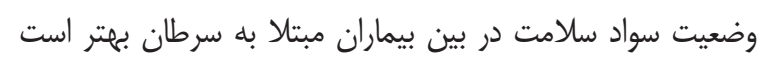
يرستاران كامهايى را جهت تضمين ارتباطات روشن و شفاف بردارند

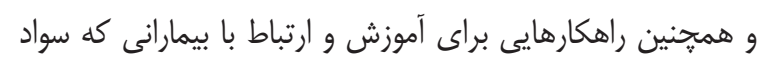

سلامت محدودى دارند، ارائه دهند.

يافتههاى يزوهش نشان دادند كه ميانگين نمره حيطه دسترسى دارنى ارته دهن

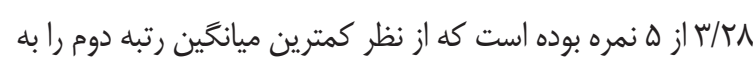
خود اختصاص داده است كه متأسفانه اين حيطه در بين بيماران مبتلا

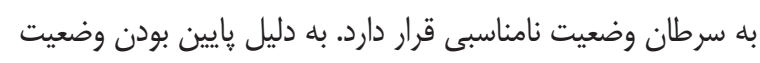
دسترسى به اطلاعات قابل فهم به نظر مىرسد كه ارتباط بين بيمار
اين يزوهش با هدف بررسى همبستخى بين رشد پِ از سانحه با

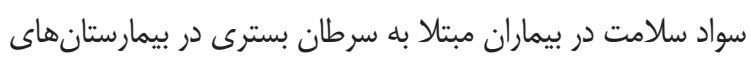

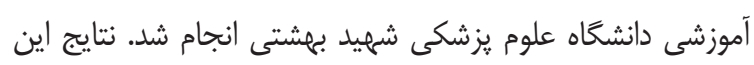

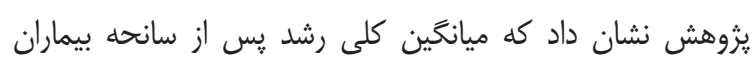

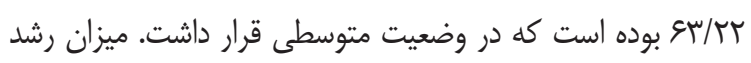

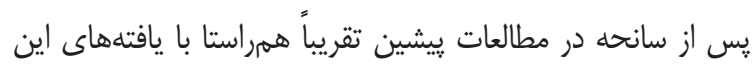

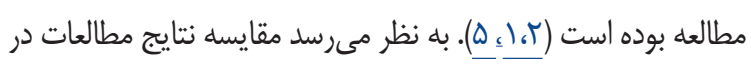

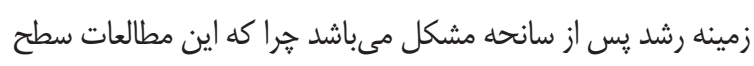

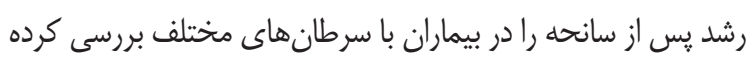

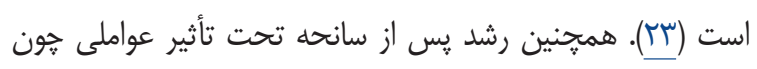

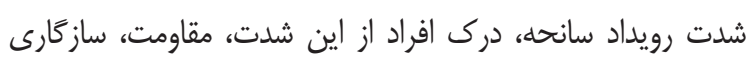
افراد، باورها و فرهنگ قرار دارد (سب، ().

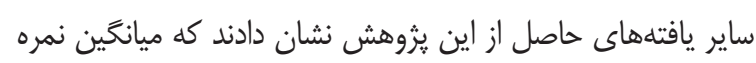

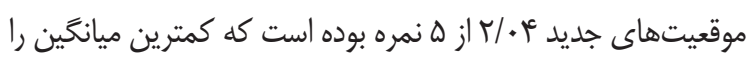

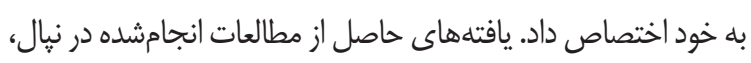

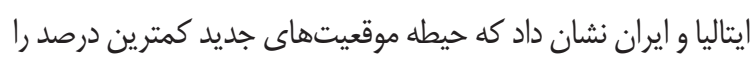

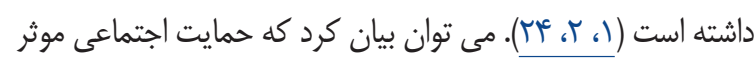

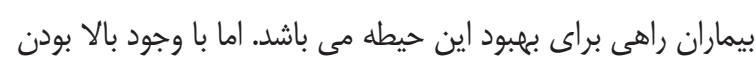

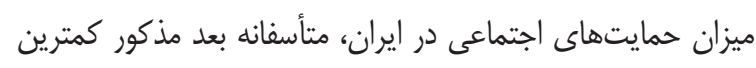

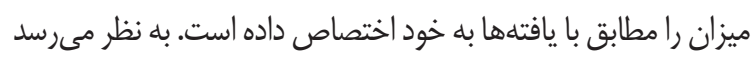

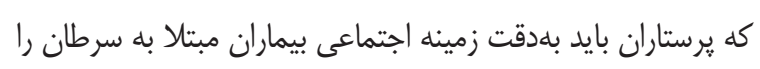

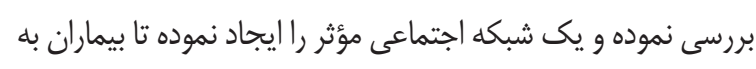

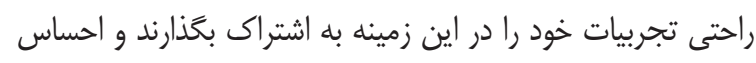
نزديكترى با ديغران داشته باشند (بآب).

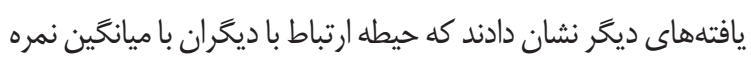

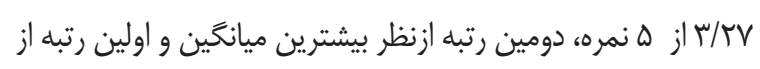

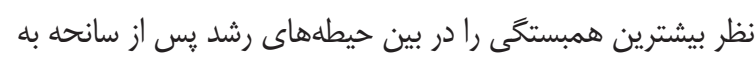

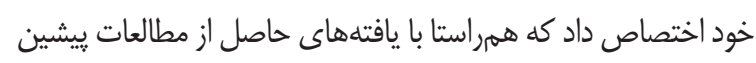

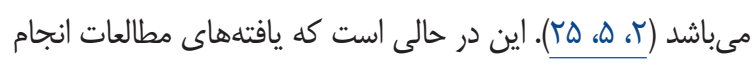

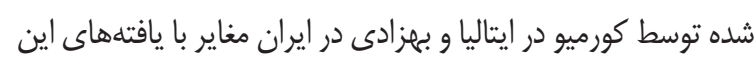

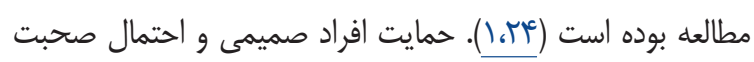

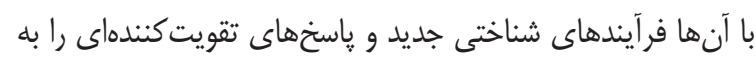

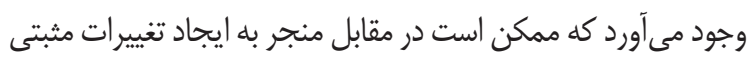

شود (ب)

عالاوه بر اين يافتههاى مطالعه نشان دادند كه حيطه قدرت شخصى

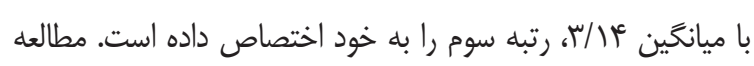


در وضعيت مطلوبى قرار دارد در حالى كه داراى كمترين همبستىى در بين حيطههاى يرسشنامه مىباشد. در مطالعه انجام شده در آمريكا

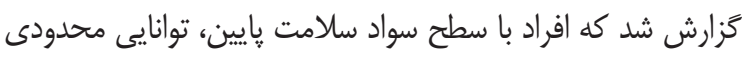
جهت دستيابى به نظام مراقبت سلامت و در نتيجه تصميمات درمانى

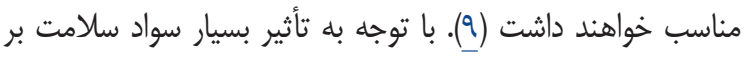

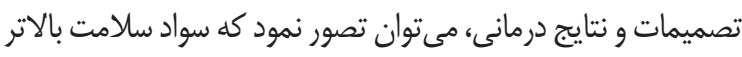

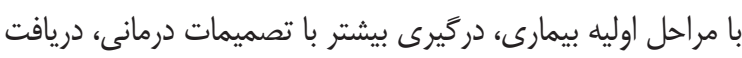
مراقبت متناسبتر همراه است (ساب).

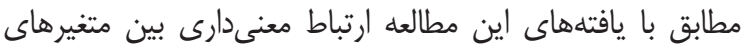

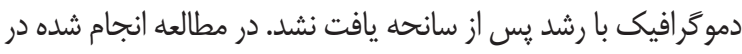

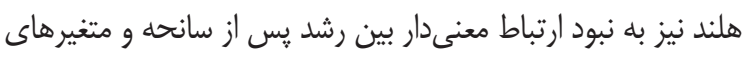

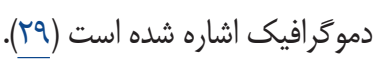
در ساير يافته ها ارتباط معنىدارى بين متغير سن بيماران با سواد

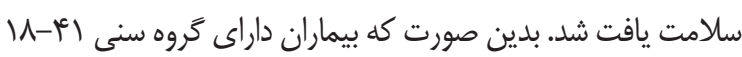
سال، سواد سلامت بالاترى نسبت به سايرين داشتند. مى بوتوان نتيجه

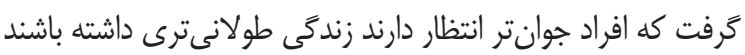

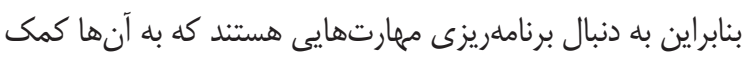

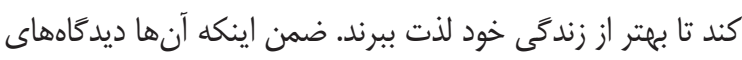
بازترى در رابطه با يادگيرى و تغيير دارند.

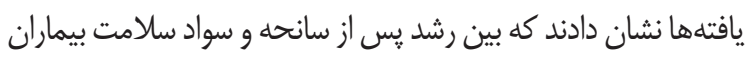

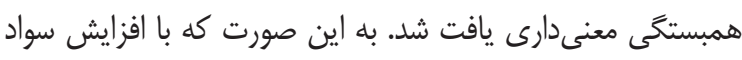

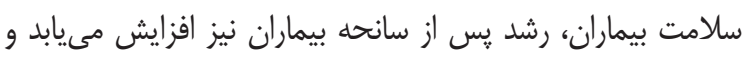

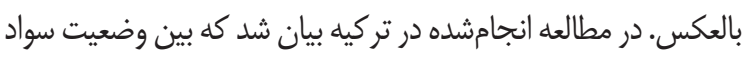

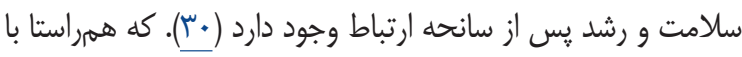

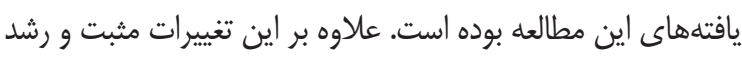

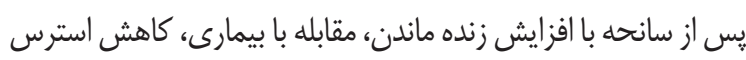

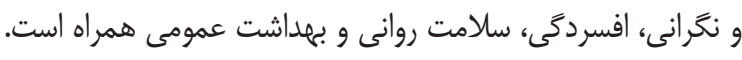

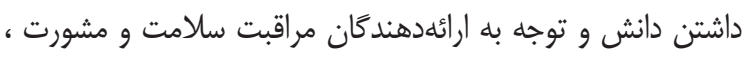

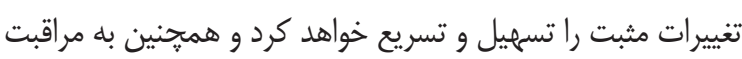

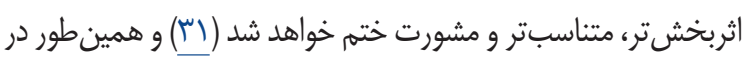

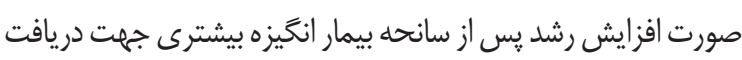

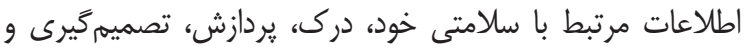

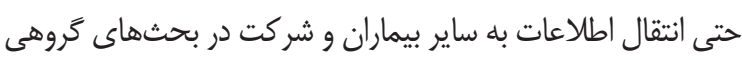

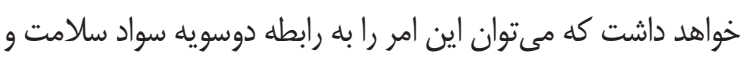
رشد يس از سانحه نسبت داد.

نتيجه كيرى متخصصان بايد به دقت زمينه اجتماعى بيماران مبتلا به سرطان را
و ارائه دهندكان در وضعيت مطلوبى قرار ندارد. علاوه بر اين ممكن

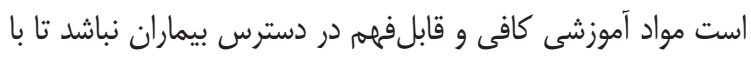

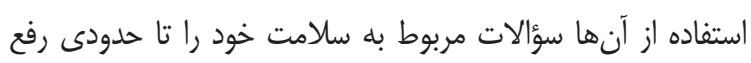

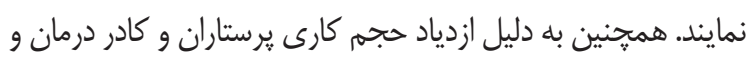
نبود وقت كافى جهت ارائه اطلاعات ارتباط بين بيمار و ارائه دهنده كارد محدود مى باشد كه خود مانعى جهت دسترسى به اطلاعات مى الهاشد.

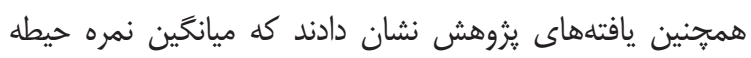

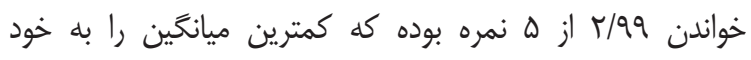

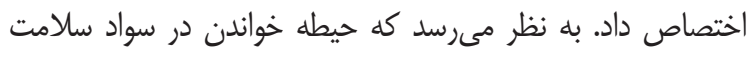

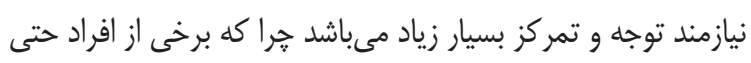

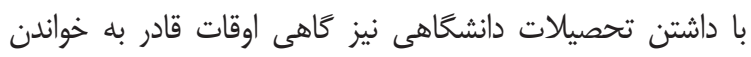

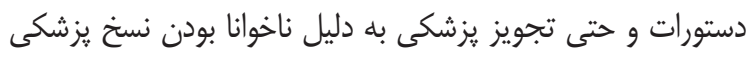

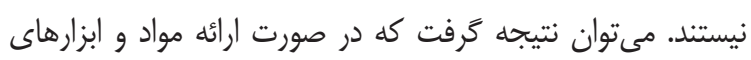

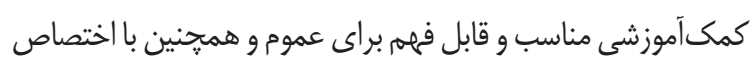

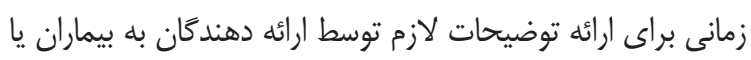

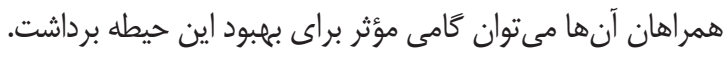

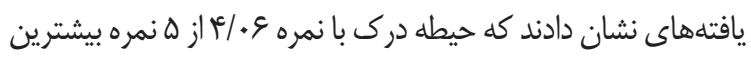

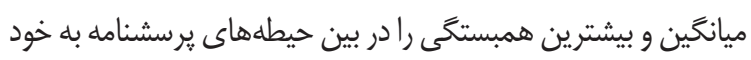

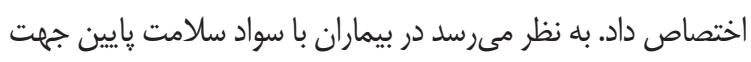

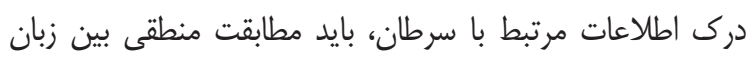

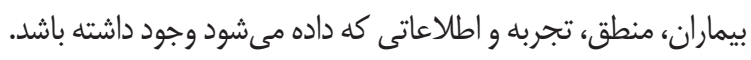

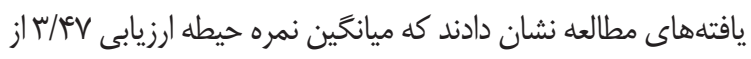

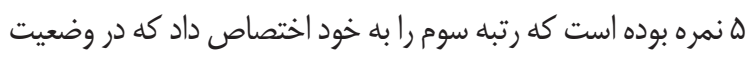

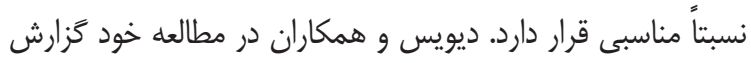

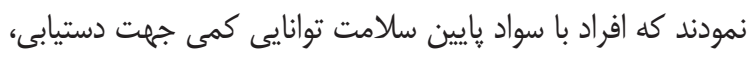
يردازش و درك اطلاعات شفاهى و كتبى دارند (بر).

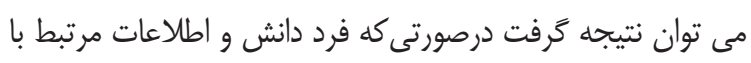

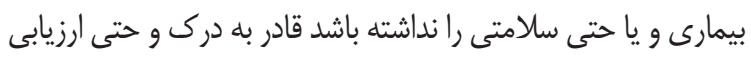

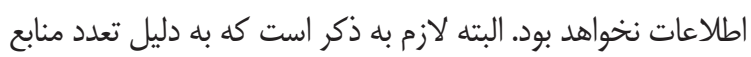

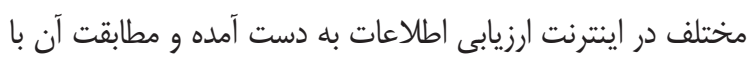

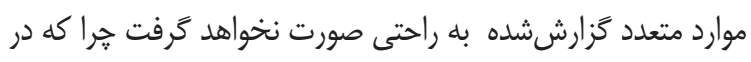

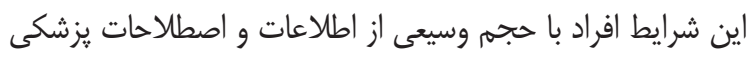

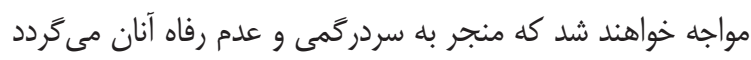

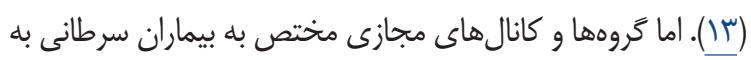

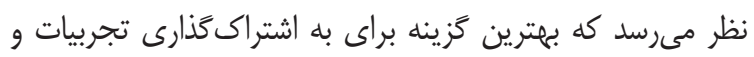

$$
\text { ارزيابى اطلاعات مىباشند. }
$$

يافتههاى مطالعه نشان دادند كه ميانخين نمره حيطه تصميمَّيرى و

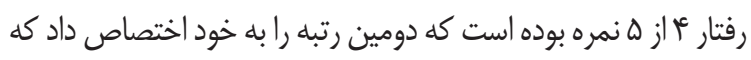


اطلاعات قابل فهم و در دسترس قرار دادن منابع اطلاعاتى گامى مؤثر بردارند.

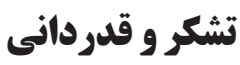

بدين وسيله از كليه افرادى كه در انجام يزوهش حاضر همكارى

$$
\text { نمودند، تشكر و قدردانى به عمل مى آيد. }
$$

\section{References}

1. Behzadi M, Rassouli M, Mojen LK, Pourhoseingholi MA, Karahroudy FA. Posttraumatic Growth and Its Dimensions in the Mothers of Children with Cancer. International Journal of Community Based Nursing and Midwifery. 2018;6 (3): 209-17.

2. Sharma A ZJ. Predictors of Post traumatic growth among breast cancer patients in Nepal. Asian Pacific Journal of Health Sciences.2017;4 (2): 9-17.

3. Sajjadi M, Rassouli M, Abbaszadeh A, Majd HA, Zendehdel K. Psychometric properties of the Persian version of the Mishel's Uncertainty in Illness Scale in Patients with Cancer. European Journal of Oncology Nursing. 2014; 18 (1):52-7.

4. Cormio C, Romito F, Viscanti G, Turaccio M, Lorusso V, Mattioli V. Psychological well-being and posttraumatic growth in caregivers of cancer patients. Frontiers in psychology. 2014; 5: 1342.

5. Heidarzadeh M, Rassouli M, Shahbolaghi FM, Majd HA, Karam A-M, Mirzaee H, et al. Posttraumatic growth and its dimensions in patients with cancer. Middle East Journal of Cancer. 2014;5 (1): 23-9.

6. Hashemi SM, Raghibi M. The Positive Role Of Structured Group Therapy On Post-Traumatic Growth Of Positive Psychological Components (PTG) In Women With Breast Cancer. Biomedical \& Pharmacology Journal. 2014;7 (2): 535.

7. Rippentrop AE, Altmaier EM, Chen JJ, Found EM, Keffala VJ. The relationship between religion/spirituality and physical health, mental health, and pain in a chronic pain population. Pain. 2005; 116 (3): 311-21.

8. Jaafari A, Dehshiri G, Sohrabi F, Najafi M. Investigate the relationship between spiritual well-being and mental health. Counseling News and Research. 2009; 8: 33-48.

9. Morris NS, Field TS, Wagner JL, Cutrona SL,

$$
\begin{aligned}
& \text { بررسى نموده و يك شبكه اجتماعى مؤثر را ايجاد نموده تا بيماران به } \\
& \text { راحتى تجربيات خود را در اين زمينه به اشتراك بخذارند و احساس } \\
& \text { نزديكترى با ديخًان داشته باشند. همجنين كادر درمان مىتوانند در } \\
& \text { زمينه حل مشكلات، دادن اطلاعات، شنيدن و نشان دادن هم دردى } \\
& \text { كام بزرگى بردارند. علاوه بر اين براى كاهش موانع سواد سلامت } \\
& \text { يرستاران مىتوانند با برقرارى ارتباط مؤثر با بيماران و ارائه دانش و }
\end{aligned}
$$

Roblin DW, Gaglio B, et al. The association between health literacy and cancer-related attitudes, behaviors, and knowledge. Journal of health communication. 2013; 18 (sup1): 223-41.

10. Treloar C, Gray R, Brener L, Jackson C, Saunders V, Johnson P, et al. Health literacy in relation to cancer: addressing the silence about and absence of cancer discussion among A boriginal people, communities and health services. Health \& social care in the community. 2013; 21 (6): 655-64.

11. Saranjit S, Lennard L. Health literacy: being able to make the most of health: National Consumer Council; 2004.

12. Arozullah AM, Lee SYD, Khan T, Kurup S, Ryan $\mathrm{J}$, Bonner $\mathrm{M}$, et al. The roles of low literacy and social support in predicting the preventability of hospital admission. Journal of general internal medicine. 2006; 21 (2): 140-5.

13. Busch EL, Martin C, DeWalt DA, Sandler RS. Functional health literacy, chemotherapy decisions, and outcomes among a colorectal cancer cohort. Cancer Control. 2015; 22 (1): 95 101.

14. Brach C, Keller D, Hernandez LM, Baur C, Dreyer B, Schyve P, et al. Ten attributes of health literate health care organizations: Institute of Medicine of the National Academies Washington, DC; 2012.

15. Nekoei-Moghadam M PS, Amiresmaili M, Baneshi M. Health Literacy and Utilization of health Services in Kerman urban Area 2011. Tolue Behdasht Journal. 2012; 11 (14):123-34.

16. Reisi M, Mostafavi F, Hasanzadeh A, Sharifirad Gh. The Relationship between Health Literacy, Health Status and Healthy Behaviors among Elderly in Isfahan. Journal of Health Systems Research. 2011;7 (4):1-12.

17. Tol A, Pourreza A, Tavasoli E, Rahimi Foroshani A. Determination of knowledge and health literacy among women with type 2 diabetes in 
teaching hospitals of TUMS. Journal of Hospital. 2012; 11 (3).

18. Peyman N, Abdollahi M. The relationship between health literacy and self-efficacy physical activity in postpartum women. Journal of Health Literacy. 2016; 1 (1): 5-12.

19. Chew LD, Bradley KA, Boyko EJ. Brief questions to identify patients with inadequate health literacy. health. 2004; 11: 12.

20. Edwards M, Wood F, Davies M, Edwards A. The development of health literacy in patients with a long-term health condition: the health literacy pathway model. BMC public health. 2012; 12 (1): 130 .

21. Tedeschi RG, Calhoun LG. The Posttraumatic Growth Inventory: Measuring the positive legacy of trauma. Journal of traumatic stress. 1996; 9 (3): 455-71.

22. Montazeri A TM, Rakhshani F,Azin A, Jahangiri K, Ebadi M, Naderimagham Sh,Soleymanian A, Sarbandi F, Motamedi A, Nghibi Sistani M, Design and psychometrics of the health literacy tool of the urban population of Iran, 18 to 65 years. . Quarterly Journal. 2014;13 (5): 599-89.

23. Rahmani A MR, Ferguson C, Golizadeh L, Zirak M, Chavoshi H. Posttraumatic growth in Iranian cancer patients. Indian J Cancer. 2012; 49: 287 -92 .

24. Cormio C, Muzzatti B, Romito F, Mattioli V, Annunziata MA. Posttraumatic growth and cancer: a study 5 years after treatment end. Supportive Care in Cancer. 2017;25(4):1087-96.

25. Heidarzadeh M DB, Gholchin M . Post-traumatic growth, hope, and depression in elderly cancer patients. International Journal of Medical Research \& Health Science. 2016; 5 (9): 455-61.

26. Karami A. The role of Daily Spiritual Experiences in the Hope and Posttraumatic Growth among Patients with Leukemia. Health, Spirituality and Medical Ethics. 2018;5(2):23-9.

27. Egan R, Liewellyn R, Wood S, Doherty J, Albert T, Walsh C, Atkinson K, Kerslake aP. The Cancer Stories Project: narratives of encounters with cancer in Aotearoa, New Zealand. PsychoOncology. 2016: 300-7.

28. Davis TC, Williams MV, Marin E, Parker RM, Glass J. Health literacy and cancer communication. CA: a cancer journal for clinicians. 2002; 52 (3): 134-49.

29. Greup SR, Kaal SE, Jansen R, Manten-Horst E, Thong MS, van der Graaf WT, et al. Posttraumatic growth and resilience in adolescent and young adult cancer patients: an overview. Journal of adolescent and young adult oncology. 2018; 7 (1): 1-14.

30. Yorulmaz H, Bayraktar S, Özdilli K. Posttraumatic growth in chronic kidney failure disease. Procedia-Social and Behavioral Sciences. 2010; 5: 2313-9.

31. Bahrami M, Taleghani F, Loripoor M, Yousefy A. Positive changes after breast cancer: A qualitative study. Journal of education and health promotion. 2015 ; 4. 